\section{KOMPASS}

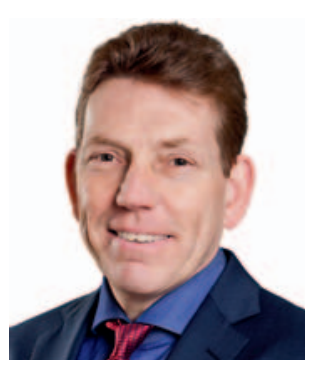

Univ.-Prof. Dr. med. Thomas Kohnen

Klinik für Augenheilkunde,

Goethe-Universität Frankfurt/M.

Die alternde Bevölkerung stellt auch die Augenheilkunde vor große Herausforderungen. Ophthalmologische Volkskrankheiten wie z.B. die altersabhängige Makuladegeneration (AMD), die Katarakt oder das Glaukom werden künftig in noch größerer Anzahl auftrennen und zu therapieren sein. Durch Früherkennung und neue Behandlungsmethoden ist es uns möglich geworden, diese Erkrankungen positiv zu beeinflussen und zu therapieren. So haben z.B. bei der AMD die Anti-VEGF-Medikationen das Feld revolutioniert, in der Glaukombehandlung führten neuartige Medikamente und minimalinvasive Glaukomchirurgie (MICS) zu schonender Drucksenkung, und bei der Kataraktchirurgie konnten durch die refraktiven Verfahren Ergebnisse erzielt werden, von denen wir vor 20 Jahren noch geträumt haben. Nehmen wir hier als ein Beispiel die Neuentwicklungen beim Linsenersatz. Bis vor wenigen Jahren noch stand bei einer Kataraktextraktion die Implantation einer Kunstlinse im Vordergrund, um so nach der Linsenextraktion die Aphakie zu beseitigen. Inzwischen wurden Intraokularlinsen (IOL) mit optischen Eigenschaften entwickelt, die nach dem Eingriff eine bessere Sehqualität erlauben [1]. So wird durch eine asphärische

\title{
Das Auge im Alter: Chancen und Herausforderungen in Behandlung und Therapie
}

IOL eine Reduktion der sphärischen Aberrationen des Auges erzielt und eine Verbesserung der optischen Qualität, besonders beim Nachtsehen, erzeugt; torische Kunstlinsen korrigieren Abbildungsfehler von Zernike-Polynomen der zweiten Ordnung [2] und schaffen so ein scharfes Bild auf der Netzhaut beim präexsistenten Hornhautastigmatismus; und alterssichtigkeitskorrigierende IOL erzeugen ein scharfes Sehen, sowohl für die Ferne als auch für den Intermediär- sowie den Nahbereich.

In dieser Ausgabe des Karger Kompass OphtHALMOLOGIE greifen mehrere Autoren das Thema «Augenerkrankungn im Alter» anhand von unterschiedlichen Krankheitsbildern und Szenarien auf: die Übersichtsarbeit gibt einen Eindruck der Möglichkeit, die Kataraktchirurgie als Glaukombehandlung in Betracht zu ziehen; Prof. Nicolas Feltgen (Göttingen) ruft zur dauerhaften Kontrolle von AMD-Patienten auf; Prof. Klaus Rohrschneider (Heidelberg) stellt wichtige aktuelle Beiträge zur Glaukomdiagnostik im Alter zusammen; und die «Erfahrung aus der Praxis» veranschaulicht besondere Herausforderungen der Linsenchirurgie in Abhängigkeit der Linsenmorphologie. Alle Arbeiten eröffnen uns neue Aspekte für die
Behandlung der immer älter werdenden Bevölkerung. Therapiekonzepte werden überdacht und auch kombiniert, wie z.B. die Linsenextraktion als Behandlung des Glaukoms, besonders bei engen Vorderabschnittsverhältnissen.

Die moderne Ophthalmologie schafft Möglichkeiten, an die vor Dekaden noch nicht zu denken war. Doch ein Wermutstropfen bleibt: Das System will auch bezahlt werden. Und hier besteht eine besondere Herausforderung für die nächsten Jahre. Eine Kostensteigerung ist nicht nur durch eine größere Anzahl an Behandlungsfällen, sondern auch durch die Vielzahl an neuen Behandlungsmethoden zu erwarten. Es bleibt also spannend. Mit Blick auf den medizinischen Fortschritt dürfen wir für unsere Patienten und die Augenheilkunde positiv in die Zukunft schauen.

Ich wünsche Ihnen viel Spaß bei der Lektüre dieser Ausgabe von Karger Kompass Ophthalmologie!

\section{Herzlichst}

Ihr

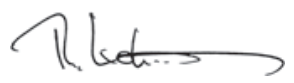

Prof. Dr. Thomas Kohnen

\section{Literatur}

1 Kohnen T: Intraokularlinsen mit besonderen Eigenschaften. Klin Monatsbl Augenheilkd 2016;233:908-909.

2 Bühren J, Kohnen T: Anwendung der Wellenfrontanalyse in Klinik und Wissenschaft. Vom irregulären Astigmatismus zu Aberrationen höherer Ordnung - Teil I: Grundlagen. Ophthalmologe 2007;104:909-925.

\section{KARGER}

Fax +497614520714 information@karger.com www.karger.com (c) 2017 S. Karger GmbH, Freiburg
Univ.-Prof. Dr. med. Thomas Kohnen, FEBO

Direktor der Klinik für Augenheilkunde

Goethe-Universität

Theodor-Stern-Kai 7, 60590 Frankfurt/M., Deutschland

kohnen@em.uni-frankfurt.de 


\section{FOKUS}

\section{Augenerkrankungen im Alter}

\section{(10) Epidemiologie}

Weltweit wie auch in Deutschland sind die drei häufigsten Augenerkrankungen im Alter die Katarakt, die altersabhängige Makuladegeneration (AMD) und das Glaukom, speziell das chronische Offenwinkelglaukom [1, 2]. Dabei kommt in Deutschland wie auch in anderen Industrienationen den beiden letztgenannten Krankheitsbildern eine besondere Bedeutung zu, da sie die häufigsten Ursachen für eine Erblindung im Alter darstellen. Die Prävalenz von Blindheit und Sehbehinderung wird weltweit auf 285 Millionen geschätzt, 82\% aller Erblindeten sind 50 Jahre alt und älter [3]. In Deutschland lag die Prävalenz von Blindheit und Sehbehinderung 2012 bei 353000, für 2030 wird eine Zunahme auf 421000 geschätzt [4]. Die Hauptursache für eine Erblindung im Alter (>65 Jahre) in Deutschland ist die AMD. Die epidemiologische Studienlage zur AMD ist in Deutschland bislang limitiert. Lediglich die Gutenberg-Gesundheitsstudie (GHS) veröffentlichte im Jahr 2014 erste Daten, die eine Prävalenz von 11,9\% für die frühe und 0,2\% für die späte AMD aufzeigten [5]. Hinsichtlich des Glaukoms geht man von etwa 520000 Neuerkrankungen in Deutschland in den Jahren 2010 bis 2015 aus. Die Zahl der Glaukomerblindungen liegt in Deutschland bei ungefähr 40000 , wozu jährlich über 1000 neu hinzukommen. Die Dunkelziffer der Glaukome wird in den Industrienationen insgesamt auf ca. 50\% geschätzt [6].

\section{Herausforderungen und Handlungsbedarf}

Eine Grundvoraussetzung für eine adäquate Behandlung und vor allem für eine Früherkennung einer Erkrankung sind gute epidemiologische Daten. Diesbezüglich ist die Situation in Deutschland im Hinblick auf die AMD [7] und das Glaukom [8] unbefriedigend, weitere Bemühungen und gesundheitspolitische Unterstützungen für eine solide Datenerfassung sollten erfolgen.

Aufgrund der demografischen Entwicklung ist mit einer steigenden Inzidenz und Prävalenz für die AMD und das Glaukom zu rechnen. Ohne verbesserte Behandlungsmöglichkeiten würden sich allein aufgrund dieser Entwicklung bis 2030 jährlich 2833 Neuerblindungen ergeben [9]. Gleichzeitig werden die in Deutschland ausgebildeten Fachärzte für Augenheilkunde den zusätzlichen Bedarf in den nächsten Jahren voraussichtlich nicht decken können. Daher sollte auch über unkonventionelle Modelle für ein Screening nachgedacht werden. Dieses ist bei den Krankheitsbildern Glaukom und AMD besonders wichtig, da es Therapieoptionen gibt, die nur bei frühzeitiger Behandlung einen Schutz vor Erblindung bieten. Hier wäre z.B. ein Screening über Telemedizin oder durch nicht-medizinisches, aber entsprechend geschultes Personal denkbar, wie es in Großbritannien bereits vor ca. 10 Jahren für die diabetische Retinopathie durch das staatliche Gesundheitssystem erfolgreich eingeführt wurde [10].

Insbesondere das Screening und die Versorgung von Sehbehinderten in Pflegeeinrichtungen stellt eine Herausforderung dar und sollte systematisch etabliert werden.

\section{Therapieoptionen}

Sowohl hinsichtlich des Glaukoms [11] als auch hinsichtlich der AMD [12] stehen uns inzwischen vielversprechende diagnostische und therapeutische Möglichkeiten zur Verfügung, die zwar die Erkrankungen nicht heilen, aber die Prognose deutlich verbessern können. Nach wie vor sind vor allem eine frühe Erkennung und eine konsequente therapeutische Begleitung und Kontrolle entscheidend, wenn es um die Verhinderung von Erblindung geht.

Zunehmend können auch genderspezifische Risikofaktoren bedacht werden [3].

Eine neue Herausforderung sind nicht selten die Überschneidungen der Therapien von verschiedenen ophthalmologischen Krankheitsbildern im Alter, wie z.B. eine operationsbedürftige Katarakt bei therapierter feuchter AMD. Hier müssen Entscheidungen hinsichtlich des sinnvollen Operationszeitpunktes der Katarakt, der möglichen Mitbehandlung der AMD und der möglicherweise intra- und postoperativ erhöhten Risiken bedacht werden [13].

\section{Literatur}

1 Klein R, Klein BEK: The prevalence of age-related eye diseases and visual impairment in aging: current estimates. Invest Ophthalmol Vis Sci 2013;54:ORSF5-ORSF13.

2 Reitmeier $\mathrm{P}$, Linkohr $\mathrm{B}$, Heier $\mathrm{M}$, et al.: Common eye diseases in older adults of southern Germany: results from the KORA-Age study. Age Ageing 2016;DOl:10.1093/ageing/afw2342016.

3 Zetterberg M: Age-related eye disease and gender. Maturitas 2016;83:19-26.

4 Deutsche Ophthalmologische Gesellschaft: Weißbuch zur Situation der ophthalmologischen Versorgung in Deutschland, September 2012. www.dog.org/wp-content/uploads/2013/03/DOG_Weissbuch_2012_ fin.pdf (letzter Zugriff: 19.12.2016).

5 Korb CA, Kottler UB, Wolfram C, et al.: Prevalence of age-related macular degeneration in a large European cohort: results from the populationbased Gutenberg Health Study. Graefe's Arch Clin Exp Ophthalmol 2014;252:1403-1411.

6 Deutsche Ophthalmologische Gesellschaft: Stellungnahme der Deutschen Ophthalmologischen Gesellschaft zur Glaukomfrüherkennung, 2015. www.dog.org/wp-content/uploads/2015/11/SN-GlaukomAugust-2015.pdf (letzter Zugriff: 19.12.2016).

7 Brandl C, Stark KJ, Wintergerst M: Epidemiologie der altersbedingten Makuladegeneration. Ophthalmologe 2016;113:735-745.

8 Oberacher-Velten I, Hoffmann E, Helbig H: Volkskrankheit Glaukome. Ophthalmologe 2016;113:746-751.

9 Ziemssen F, Röck D: Zukunft der Augenheilkunde? Zahlen und Fakten. Z prakt Augenheilkd 2013;34:457-461.

10 The Heart of England Diabetic Retinopathy Screening Centre. www.retinalscreening.co.uk/about-us/ (letzter Zugriff 19.12.2016).

11 Balendra SI, Shah PA, Jain M, et al.: Glaucoma: hot topics in pharmacology. Curr Pharm Des 2016;29 [Epub ahead of print].

12 Mantel I: Age-related macular degeneration - a challenge for public health care. Ther Umsch 2016;73:79-83.

13 Hahn P, Yashkin AP, Sloan FA: Effect of prior anti-VEGF injections on the risk of retained lens fragments and endophthalmitis after cataract surgery in the elderly. Ophthalmology 2016;123:309-315.

PD Dr. Anja Liekfeld, Klinikum Ernst von Bergmann, Potsdam

Chefärztin der Klinik für Augenheilkunde 